\title{
Vitamin D Deficiency: A common problem worldwide
}

Narayan B. Mahotra

Author Info:

Dr. Narayan B. Mahotra, MD

Associate Professor, Department of Clinical Physiology, Maharajgunj Medical Campus, Institute of Medicine, Tribhuvan University, Kathmandu.

\section{Correspondence:}

Email/Contact:

narayanmahotra@gmail.com

\begin{abstract}
Sunlight exposure is the most important source of vitamin D for human beings. We can get vitamin $D$ from other sources such as diet and dietary supplements as well. Human body synthesizes most of the vitamin $D$ from exposure to direct Ultraviolet B (UVB) sunlight. Regular, short periods of UVB exposure during the summer months are enough to get required vitamin $D$ for most people. Sunscreen might block the vitamin D synthesis process in skin. Solar ultraviolet B radiation (wavelength, 290 to $315 \mathrm{~nm}$ ) penetrates the skin and converts7-dehydrocholesterol to provitamin D3, which is rapidly converted to vitamin D3. Excessive exposure to sunlight does not cause vitamin D3 intoxication but excess oral intake can cause intoxication. Therefore, we should be careful while taking dietary supplements of vitamin D.
\end{abstract}

Regardless of the good sunlight exposure, surprisingly, the blood level of vitamin $\mathrm{D}$ is found to be low in people of South Asian countries including Nepal. No practice of sun bath and all the time body coverage with clothes could be the possible reason of low vitamin $D$ level in this region of the world. Another possibility is normal reference value of vitamin $D$ level might be low in South Asians compared to western countries. So, large scale research including all required parameters can solve these concerns and queries.

Keywords: Sunlight, Ultraviolet B, Vitamin-D

\section{QR Code}

[2) View PDF

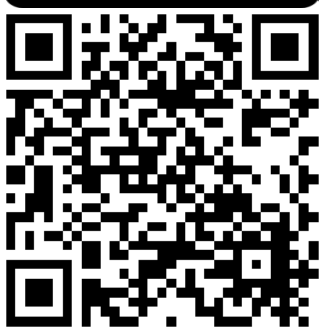

Scan Me for Full Text

\section{Article Info}

Received: 13 September 2020; Accepted: 9 december 2020; Published Online: 10 december 2020 How to cite this article in Vancouver Style?

Mahotra NB. Vitamin D Deficiency: A common problem worldwide..Europasian J Med Sci.2020;2(2): 145-147. https://doi.org/10.46405/ejms.v2i2.184

\section{Disclaimer:}

Conflict of Interest: None Declared

Source of Support: Nil

Copyright: $(2020$ by author(s). This is an open access article distributed under the terms of the Creative Commons Attribution International License 4.0 @() (1) which permits unrestricted use, distribution, and reproduction in any medium, provided the original work is properly cited.

Publisher's Note:

The Europasian Journal of Medical Sciences (EJMS) (www.europasianjournals.org) is an official Journal of Nirvana Psychosocial Care Center \& Ressearch Institute (www.nirvanapscc.com). The Journal as well as publisher remain neutral with regards to any jurisdictional claims in any published articles, its contents and the institutional affiliations of the authors. 


\section{INTRODUCTION}

Vitamin $D$ is one of the important vitamins which is essential for our body development and maintenance of homeostasis. It is mainly obtained from the sunlight exposure to skin and also from the diet but it is difficult to obtain enough vitamin $\mathrm{D}$ from diet alone.

Vitamin D is not sufficiently available in dietary sources. We can get vitamin D from some fish products such as sardines, mackerel, salmon tuna, and fortified margarines, egg yolks and red meat. These vitamin $D$ containing dietary sources can be recommended as part of a healthy balanced diet. ${ }^{1}$ Cholecalciferol which comes from the skin or from the diet, is converted in the liver to 25-hydroxcholecalciferol, which is used to determine a patient's vitamin $D$ status. 25-hydroxycholecalciferol is then converted in the kidneys by the enzyme $1 \alpha$-hydroxylase to its active form, 1, 25-dihydroxycholecalciferol. The 1,25 dihydroxycholecalciferol, also called Calcitriol is the hormonal form of vitamin D which is required for intestinal absorption of calcium. ${ }^{2}$ The rate of secretion of 1, 25-dihydroxycholecalciferol by kidneys is strictly regulated by blood parathyroid hormone level as well as blood calcium and phosphorus levels. Fibroblast growth factor 23 is another important regulating factor which is secreted from the bone. It facilitates sodiumphosphate co-transports in the cells of kidneys and small intestine and also inhibits the synthesis of 1, 25-dihydroxycholecalciferol. The Calcitriol increases the rate of absorption of calcium from the renal tubules and of calcium and phosphorus from the intestine. ${ }^{3}$

Black people who have pigmented skin and people who have less sunlight exposure are at risk of vitamin $D$ deficiency. Skin concealing garments or use of sunscreen prevents the ultraviolet light exposure leading to vitamin D deficiency. Multiple, short interval pregnancies and elderly or housebound people, strict vegetarians or consumption of high phytate containing diet such as wheat bread, malabsorption syndrome and use of certain drugs such as phenytoin, phenobarbitone, carbamazepine rifampicin, cholestyramine and antiretroviral drugs are other important risk factors for vitamin $D$ deficiency. ${ }^{3}$

In United Kingdom, there is increasing number of reported cases of rickets in children and Osteomalacia in adults which are resulted from vitamin $D$ deficiency in both children and adult population. $^{3}$ Three- quarters of teens and adult populations are deficient in vitamin D in United States of America. The vitamin D deficiency might be associated with wide range of clinical problems such as heart disease, diabetes and even cancer. ${ }^{1}$

The prevalence of hypovitaminosis $D$ in Nepal is about $74.1 \%$, which is similar to that recorded by Mansour et al. in Saudi Arabia where $72.55 \%$ vitamin D deficiency was reported. An Indian study by J. Basu Devan et al. reported a prevalence of vitamin D deficiency to be $62.2 \%$ among children and adolescents. Similarly, a study done in Turkey showed an overall prevalence of $40 \%$ but the deficiency prevalence was much higher in adolescent girls (64.8\%). Another study conducted by Shrestha et al. in Lalitpur Nepal, have reported the prevalence of vitamin $D$ deficiency to be $78.2 \% .{ }^{4} \mathrm{~A}$ study was done in infants and mothers showed that there is a tradition of sunbathing for new born baby in Nepal. Outdoor breast feeding is a common practice in some areas of Nepal which provides frequent and sufficient sun light exposure to infant. It could be the cause of good vitamin D status in Nepali infants. ${ }^{5}$

\section{Clinical manifestations:}

Vitamin D has skeletal as well as extra-skeletal roles. In terms of extra-skeletal functions, it has a role in reducing obesity and preventing diabetes mellitus, cancer, cognitive impairment and dementia. Vitamin $D$ has regulatory functions in innate immunity as well. ${ }^{6}$ so; it might reduce the risk of infections including SARS COV 2.

In terms of skeletal functions, vitamin $D$ has a role in bony development. Hypovitaminosis D can lead to rickets in children and osteomalacia in adults. Rickets is a disease of the growing bones, characterized by the failure of normal mineralization, seen predominantly at the growth plates, resulting in softening of the bones and development of deformities. ${ }^{8}$ It is manifested as

legs bowing, knock knees, costochondral junction swelling, softening skull with frontal bossing, delayed closure of fontanels, delayed tooth eruption. ${ }^{8}$ Chronic unexplained bone pain, muscular weakness particularly seen while climbing stairs, waddling gait and delayed walking are other clinical manifestations of hypovitaminosis D. Hypocalcaemic tetany, seizures commonly seen in infants, decreased growth rate and cardiomyopathy in infants can also be manifested in vitamin D deficiency. ${ }^{5,8}$ 
Osteomalacia is an adult counterpart of rickets. It is a disease where the organic matrix of the bone fails to calcify properly. There is failure to replace the turnover of $\mathrm{Ca}++$ and phosphates in the organic matrix of the bone. As a result, bone mineralization suffers. It is commonly found in women. It is manifested as bone pain, muscle weakness, tetany may manifest as carpopedal spasm and facial twitching and spontaneous fracture may occur in spine and may result in kyphosis. ${ }^{8}$

Hypovitaminosis D is associated with the presence and severity of depressive disorders. Low serum level vitamin $D$ is likely to be found in people with either remitted or current depression. ${ }^{7}$ In spite of these manifestations, Vitamin D deficiency has an association with anorexia nervosa and bulimia nervosa which are commonly seen in women. ${ }^{6}$

\section{Preventive measures:}

Children and females are at high risk of developing hypovitaminosis D. So, primary preventive measures should focus on them. Females and children with poor diets or insufficient calcium in the diet, exclusively breast fed babies from the age of six months or if the infant has not started solid foods are very much prone to develop vitamin D deficiency. Disabled people or children, people who spend limited time outside home, black people as their skin cannot synthesize sufficient amount of vitamin $D$ and people or children who are taking anticonvulsant medications such as phenytoin, phenobarbitone, carbamazepine can easily develop hypovitaminosis D. So, we should advice the high risk group people about safe sunlight exposure, dietary intake of vitamin $D$ and supplements of vitamin D. We should suggest the people to stay in sun light without wearing full body covering garments. In developing countries like Nepal, people have practice staying in sun light but they cover whole body parts with thick garments so that the sun light is blocked to get into the skin. So, despite sufficient outdoors stay, they develop vitamin D deficiency. Knowledge should be disseminated to them regarding the vitamin $D$ containing diets. Fish, red meat and egg yolks contain good amount of vitamin D. In market, vitamin D supplements are available which can be recommended to high risk people of developing vitamin D deficiency. ${ }^{8,9}$

\section{CONCLUSION}

Vitamin D deficiency is highly prevalent worldwide. It is more commonly found in children and female population. Children with poor diet, exclusively breast fed babies from the age of six months or if the infant has not started solid foods are at high risk of developing hypovitaminosis D. Lack of sunlight exposure, low intake of vitamin $D$ in the diet, wearing skin concealing garments, increasing use of sunscreen, short interval pregnancies and malabsorption can lead to vitamin D deficiency in females. Safe and sufficient sunlight exposure, dietary intake of vitamin $D$ and supplements of vitamin D can prevent developing hypovitaminosis D.

\section{REFERENCES}

1. Ginde AA, Liu MC, Camargo CA. Demographic differences and Trends of vitamin D Insufficiency in the US population. Arch Intern Med.2009; 169 (6): 626-632. https://doi.org/10.1001/ archinternmed.2008.604

2. Holick M. Vitamin D Deficiency. N Engl J Med.2007; 357:266-81. https://doi.org/10.1056/NEJMra070553

3. NHS. Vitamin D deficiency - Clinical guidelines for adults.2015

4. Regmi S, Regmi AP, Adhikari S, Shakya D. Prevalence of vitamin $D$ deficiency/insufficiency among children and adolescents. JCMC. 2017;7(19):11-15. https:// doi.org/10.3126/jcmc.v7i1.17363

5. Haugan J, Ulak M, Chandyo RK, Henjum S, ThorneLyman AL, Ueland PM et al. Low Prevalence of Vitamin D Insufficiency among Nepalese Infants Despite High Prevalance of Vitamin D Insufficiency among Their Mothers. Nutrients.2016; 8,825. https:// doi.org/10.3390/nu8120825

6. Tasegian A, Curcio F, Ragione LD, Rossetti F, Cataldi S, Codini $M$ et al. Hypovitaminosis D3, Leukopenia and Human Serotonin Transoporter polymorphism in Anorexia Nervosa and Bulimia Nervosa. Hindawi publishing corporation. Mediators of Inflammation.2016. http.//dx.doi. org/10.1155/20168046479.

7. Milaneschi $Y$, Hoogendijk W, Lips $P$, Heijboer AC, Shoevers $R$, Hemert A $M$, at al. The association between low vitamin $D$ and depressive disorders. Molecular Pychiatry.2014; 19, 444-451. https://doi. org/10.1038/mp.2013.36

8. Colledge N.R, Walker B. R, Ralston S.H. Davidson's Principles and Practice of Medicine. $21^{\text {st }}$ ed. London: Elsevier; 2010. Chapter 25, musculoskeletal disease; p. 1121-23.

9. NICE guidance [NG34]. Sunlight exposure: risks and benefits.2016 